\section{IN VIVO DEMETHYLATION-MEDIATED REVERSAL OF TUMOR CELL-INTRINSIC CGAS SILENCING IMPROVES THE EFFICACY OF STING AGONIST THERAPY}

${ }^{1}$ Rana Falahat*, ${ }^{1}$ Patricio Perez-Villarroel, ${ }^{1}$ Anders Berglund, 'Shari Pilon-Thomas, ${ }^{2}$ Glen Barber, ${ }^{1}$ James Mule. ${ }^{1}$ Moffitt Cancer Center, Tampa, FL, United States; ${ }^{2}$ University of Miami, Miami, FL, United States

Background While STING-activating agents have shown limited efficacy in early phase clinical trials, multiple lines of evidence suggest the importance of the so far unappreciated tumor cell-intrinsic STING function in antitumor immune responses. Accordingly, we have shown that although there is a widespread impairment of STING signaling among human melanomas, its restoration through epigenetic reprogramming can augment antigenicity and $\mathrm{T}$ cell recognition of melanoma cells. ${ }^{2}$ In this study, we determined if rescue of tumor cellintrinsic STING signaling using a DNA methyltransferase inhibitor can improve the therapeutic efficacy of a STING agonist in mouse models of melanoma.

Methods We subjected three distinct murine melanoma cell lines (B16-F10, B16-ISG and Yumm1.7) to treatment with 5aza-2'-deoxycytidine (5AZADC) and evaluated their activation of STING following stimulation with the STING agonist ADU-S100. Using a B16-F10 subcutaneous model, we assessed the effect of 5AZADC treatment on the efficacy of intratumorally administered ADU-S100 in STINGgt/gt mice. Additionally, we performed mechanistic studies using T-cell depletion experiments as well as phenotypic and gene expression profiling.

Results We observed reconstitution of cGAS in all three 5AZADC-pretreated cell lines as well as up to a 46-fold increase in induction of IFN-beta $(\mathrm{p}<0.001)$ and a 4.5 -fold increase in MHC class I surface expression $(p<0.01)$ compared to untreated controls following stimulation with ADUS100. In B16-F10 tumor-bearing mice, while treatment with a combination of 5AZADC plus ADU-S100 resulted in a marked increase in Ifnb1 transcripts within tumors $(\mathrm{p}<0.001)$, it significantly delayed tumor growth compared to treatment with ADU-S100 alone ( $p=0.0244$ on day 22). Antibody-mediated depletion studies in mice receiving the combination therapy further indicated that this antitumor activity depends on the generation of functional tumor antigen-specific CD8 $+\mathrm{T}$ cells $(\mathrm{p}=0.0111$ on day 22); however, tumor growth remained unaltered by the depletion of CD4+ T cells.

Conclusions We show that reversal of methylation silencing of cGAS in murine melanoma cell lines using a clinically available DNA methylation inhibitor can improve agonist-induced STING activation and type I IFN induction, which in tumorbearing mice is capable of inducing tumor regression through a CD8 $+\mathrm{T}$ cell-dependent immune response. These findings not only provide mechanistic insight into how STING signaling dysfunction in tumor cells can contribute to impaired responses to STING agonist therapy, but also suggest, depending on tumor cell-intrinsic STING signaling status, its pharmacologic restoration should be considered for improving therapeutic efficacy of STING agonists in future clinical studies.

Acknowledgements

Funding: NCI P50 CA168536, Cindy and Jon Gruden Fund, Chris Sullivan Fund, V Foundation, Dr. Miriam and Sheldon G. Adelson Medical Research Foundation.

\section{REFERENCES}

1. Falahat R, Perez-Villarroel P, Mailloux AW, Zhu G, Pilon-Thomas S, Barber GN, Mulé JJ. STING signaling in melanoma cells shapes antigenicity and can promote antitumor T-cell activity. Cancer Immunol Res 2019;7(11):1837-48.

2. Falahat $R$, Berglund $A$, Putney RM, Perez-Villarroel $P$, Aoyama S, Pilon-Thomas $S$, Barber GN, Mulé JJ. Epigenetic reprogramming of tumor cell-intrinsic STING function sculpts antigenicity and T cell recognition of melanoma. PNAS 2021;118(15)

http://dx.doi.org/10.1136/jitc-2021-SITC2021.760 